OPEN ACCESS

Edited by:

Binquan Luan,

IBM Research, United States

Reviewed by:

Xiyun Guan,

Illinois Institute of Technology,

United States

Hai-Chen Wu,

University of Chinese Academy

of Sciences, China

*Correspondence:

Yi-Lun Ying

yilunying@ecust.edu.cn

Yi-Tao Long

yitaolong@nju.edu.cn

${ }^{\dagger}$ These authors have contributed equally to this work

Specialty section: This article was submitted to

Nanoscience,

a section of the journal

Frontiers in Chemistry

Received: 12 June 2019

Accepted: 11 July 2019

Published: 31 July 2019

Citation:

Li M-Y, Wang Y-Q, Lu Y, Ying Y-L and Long Y-T (2019) Single Molecule

Study of Hydrogen Bond Interactions Between Single Oligonucleotide and

Aerolysin Sensing Interface.

Front. Chem. 7:528.

doi: $10.3389 /$ fchem.2019.00528

\section{Single Molecule Study of Hydrogen Bond Interactions Between Single Oligonucleotide and Aerolysin Sensing Interface}

\author{
Meng-Yin $\mathrm{Li}^{1+}$, Ya-Qian Wang ${ }^{1 \dagger}$, Yao Lu ${ }^{1}$, Yi-Lun Ying ${ }^{1,2 *}$ and Yi-Tao Long ${ }^{1,2 \star}$ \\ ${ }^{1}$ School of Chemistry and Molecular Engineering, East China University of Science and Technology, Shanghai, China, ${ }^{2}$ State \\ Key Laboratory of Analytical Chemistry for Life Science, School of Chemistry and Chemical Engineering, Nanjing University, \\ Nanjing, China
}

The aerolysin nanopore displays a charming sensing capability for single oligonucleotide discrimination. When reading from the electrochemical signal, stronger interaction between the aerolysin nanopore and oligonucleotide represent prolonged duration time, thereby amplifying the hidden but intrinsic signal thus improving the sensitivity. In order to further understand and optimize the performance of the aerolysin nanopore, we focus on the investigation of the hydrogen bond interaction between nanopore, and analytes. Taking advantage of site-direct mutagenesis, single residue is replaced. According to whole protein sequence screening, the region near K238 is one of the key sensing regions. Such a positively charged amino acid is then mutagenized into cysteine and tyrosine denoted as $\mathrm{K} 238 \mathrm{C}$, and $\mathrm{K} 238 \mathrm{Y}$. As $(\mathrm{dA})_{4}$ traverses the pores, K238C dramatically produces a six times longer duration time than the WT aerolysin nanopore at the voltage of $+120 \mathrm{mV}$. However, K238Y shortens the dwell time which suggests the acceleration of the translocation causing poor sensitivity. Referring to our previous findings in $\mathrm{K} 238 \mathrm{G}$, and $\mathrm{K} 238 \mathrm{~F}$, our results suggest that the hydrogen bond does not dominate the dynamic translocation process, but enhances the interaction between pores and analytes confined in such nanopore space. These insights give detailed information for the rational design of the sensing mechanism of the aerolysin nanopore, thereby providing further understanding for the weak interactions between biomolecules and the confined space for nanopore sensing.

\section{Keywords: single-molecule interface, oligonucleotide, nanopore, hydrogen bond, nanoconfinement}

\section{INTRODUCTION}

The biological nanopore is a promising sensing tool for single molecule detection under an external ionic flux (Kasianowicz et al., 1996; Wang et al., 2013a; Cao and Long, 2018). Due to its high sensing ability for the molecules in nanometer scale, the biological nanopore has been developing especially in life science over the past three decades (Stefureac et al., 2006; Gu and Shim, 2010; Pastoriza-Gallego et al., 2011; Wen et al., 2011; Wang et al., 2015). Such a single-molecule sensing interface originating from a single protein provides a well-confined nano-space for restricting a single entity reading from electrochemical signal (Bayley and Cremer, 2001; Deamer and Branton, 2002; Ying et al., 2018). In order to optimize the current and temporal 
resolution for different individuals, various membrane proteins, and toxins have been studied as nanopores, such as $\alpha$-hemolysin (Kasianowicz et al., 1996; Ying et al., 2013), MspA (Butler et al., 2008), aerolysin (Cao et al., 2016a), phi29 DNA-packaging nanomotor (Wendell et al., 2009), SP1 (Wang et al., 2013b), OmpG (Fahie et al., 2015), NfpAB (Singh et al., 2012), ClyA (Soskine et al., 2012), FhuA (Mohammad et al., 2012), and CsgG (Brown and Clarke, 2016). Volume restriction and dynamic interaction between analyte and nanopore are considered as the two dominant factors determining the sensitivity and selectivity of the biological nanopore sensing (Ying et al., 2018). The confinement of nanopore accommodates the single analyte for the further characteristic interactions inside the nanopore, leading to the distinguishable ionic signature. For example, the dsDNA and proteins require large nanopore $(\mathrm{d}>2 \mathrm{~nm})$ such as ClyA (Soskine et al., 2012), phi29 (Wendell et al., 2009), and FhuA (Mohammad et al., 2012) for efficient non-covalent interactions, while the ssDNA, peptide and small polymers ask small nanopores ( $<2 \mathrm{~nm}$ ) such as MspA (Butler et al., 2008), CsGg (Brown and Clarke, 2016), and aerolysin (Cao et al., 2016b) for better pore-analyte interactions. In recent years, due to the special geometric structure with long $\beta$-barrel and many charged amino acid residues inside the pore lumen, aerolysin nanopore exhibited charming capability for molecule sensing, such as the discrimination of DNA or peptide with different lengths (Cao et al., 2016a; Wang et al., 2017; Piguet et al., 2018), direct readout of single molecule size of PEG and single nucleobase variations in an oligonucleotides (Baaken et al., 2015; Cao et al., 2017), even real-time monitoring of methylcytosine under serum condition (Yu et al., 2017).

As the single analyte driven inside the nanopore, the sensing region of aerolysin is responsible for most of the interaction for the whole duration. For example, our recent studies have shown that K238 site of aerolysin nanopore has sensitive interactions with oligonucleotide (Cao et al., 2018). Moreover, the replacement of positively charged lysine in 238 site with negatively charged Glutamic acid (K238E) prolongs the translocation time about 20 times for negatively charged $(\mathrm{dA})_{4}$ comparing to that of wild-type (WT) aerolysin (Wang et al., 2018a). The electrostatic repulsion between K238E and oligonucleotides induces the back and forth motion of $(\mathrm{dA})_{4}$, resulting in current variations during the single blockage event. Other mutations such as $\mathrm{K} 238 \mathrm{~F}$ and $\mathrm{K} 238 \mathrm{G}$ also demonstrate that K238 site greatly contributed to the temporal resolution for oligonucleotides detection due to the sensitive non-covalent interactions (Wang et al., 2018b). However, the questions still remain for the dominated type of non-covalent interaction inside aerolysin nanopore as sensing the oligonucleotide.

Among the non-covalent interactions, hydrogen bond is typical and considered in the following content. We further designed two mutant aerolysin nanopores (K238C and K238Y) to study the role of hydrogen-bond interaction at 238 site for the translocation behaviors of oligonucleotide (Figures 1A,B). Compared to $\mathrm{K} 238 \mathrm{~F}$ and $\mathrm{K} 238 \mathrm{G}$ in our previous study (Wang et al., 2018b), K238Y and K238C possess hydroxyl, and sulfhydryl group on the amino acid residues, respectively. In principle, these two mutations could site-directly increase the possibility for forming hydrogen bonds during the dynamic interaction process with oligonucleotides (Luscombe et al., 2001). The results show that $\mathrm{K} 238 \mathrm{C}$ mutant nanopore produces extremely long blockage duration especially at the applied voltage of $+120 \mathrm{mV}$, which is approximately six times longer than WT aerolysin, and about $\sim 1.3 \mathrm{~ms}$ longer than K238G mutant. Furthermore, the duration of oligonucleotide with $\mathrm{K} 238 \mathrm{Y}$ is about $\sim 1 \mathrm{~ms}$ longer than that inside K238F (Figure 1C). Therefore, the hydrogen bond is one of the crucial factors for the oligonucleotide-pore interactions. This study could guide us to sophisticate design the sensing interface of aerolysin for further practical application on microRNA detection, DNA damage, epigenetic modifications, and even examine the activity of nucleases.

\section{MATERIALS AND METHODS}

\section{Chemicals and Reagents}

Trypsin-agarose, trypsin-EDTA, decane (anhydrous, $\geq 99 \%$ ), $\mathrm{Na}_{3} \mathrm{PO}_{4}, \mathrm{HCl}, \mathrm{NaCl}$, and imidazole are purchased from SigmaAldrich Co., Ltd. (St. Louis, MO, USA). 1, 2-diphytanoylsnglycero-3-phosphocholine (chloroform, $\geq 99 \%$ ) is purchased from Avanti Polar Lipids, Inc. (Alabaster, Al, USA). All polynucleotides samples are synthesized and HPLC-purified by Sangon Biotech Co., Ltd. (Shanghai, China). All reagents and materials are of analytical grade. Yeast extract and peptone are purchased from OXOID Co., Ltd. (Basingstoke, UK). Glycerinum is purchased from Amresco, Inc. (Atlanta, GA, USA). IPTG is purchased from Inalco SpA, Inc. (Milano, Italy). BL21 [DE3] pLysS E. coli is purchased from TIANGEN Co., Ltd. (Beijing, China). The pET22b-proaerolysin plasmid are synthesized, and HPLC-purified by Genewiz, Inc. (Suzhou, China). All solutions are prepared using ultrapure water (18.2 $\mathrm{M} \Omega \mathrm{cm}$ at $25^{\circ} \mathrm{C}$ ) from a Milli-Q system (Billerica, MA, USA).

\section{Proaerolysin Production}

The preoaerolysin productions of $\mathrm{K} 238 \mathrm{C}$ and $\mathrm{K} 238 \mathrm{Y}$ are according to the previous studies (Iacovache et al., 2011; Wang et al., 2018a). Note that mercaptoethanol was added to the K238C aerolysin nanopore to protect the cysteine from oxidation.

\section{Single Molecule Measurement}

The formation of mutant aerolysin nanopore is described in our previous study (Wang et al., 2018a). Both compartments of the recording chamber contain $1.0 \mathrm{~mL}$ of $1.0 \mathrm{M} \mathrm{KCl}, 10 \mathrm{mM}$ Tris, $\mathrm{pH}$ 8.0, with $1.0 \mathrm{mM}$ EDTA. The potential is applied using $\mathrm{Ag} / \mathrm{AgCl}$ electrodes. About $1.0 \mu \mathrm{L}$ monomeric aerolysin $(\sim 1.5 \mu \mathrm{g} / \mathrm{mL})$ is added to the cis chamber to form the pore. The oligonucleotide is added to the cis compartment to a final concentration of $2.0 \mu \mathrm{M}$. All the nanopore experiments are conducted at $24 \pm 2^{\circ} \mathrm{C}$. All nanopore experiments for each mutant aerolysin were performed at least 3 separated measurements.

\section{Data Acquisition and Analysis}

The current recordings are performed with a patch clamp amplifier (Axon 200B equipped with a Digidata 1440A A/D converter, Molecular Devices, USA). The amplified signal (arising from the ionic current passing through the pore) is sampled 


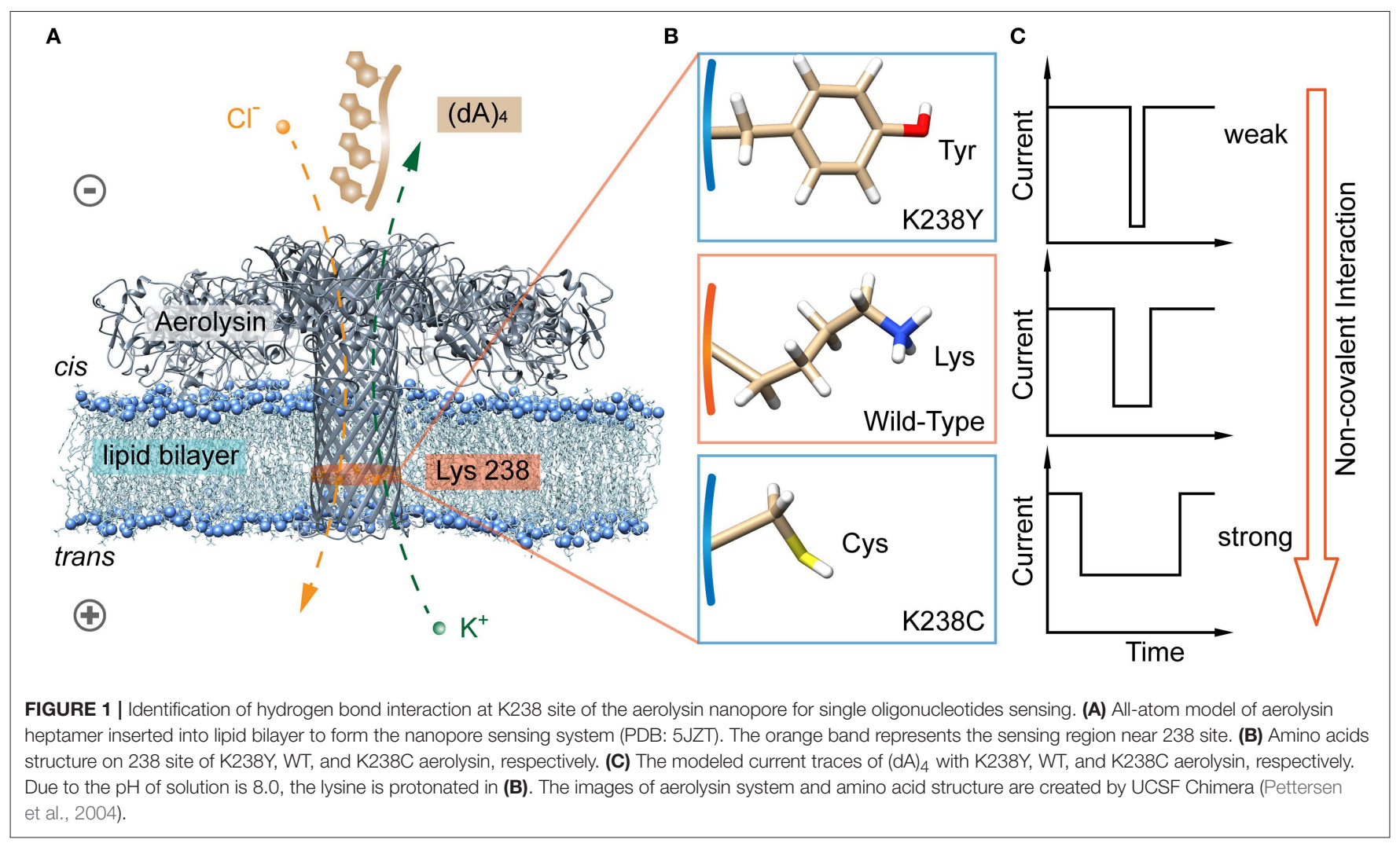

at $100 \mathrm{kHz}$ and low-pass filtered at $5 \mathrm{kHz}$ through the Clampex 10.7 software (Molecular Devices, USA). The data analysis is performed by using Mosaic software (Balijepalli et al., 2014; Forstater et al., 2016), and Origin-Lab 8.0 (Origin-Lab Corporation, Northampton, MA).

\section{RESULTS AND DISCUSSIONS}

The aerolysin produced by proaerolysin is able to self-assemble and oligomerize as a heptameric ring-like structure that inserts into the membrane to form a nanoscale pore (Iacovache et al., 2016). As illustrated in Figure 1A, the orange-banded region that near the positively charged lysine-238 (K238) site is considered as one of the sensing regions due to its relatively small diameter and special potential distribution under an applied voltage (Wang et al., 2018b). To estimate the influences of hydrogen bonds between the amino acid residues inside the pore, and oligonucleotides on its translocation behaviors, the positively charged lysine is mutated into neutral amino acid residues of cysteine (C), and tyrosine (Y) that owns sulfhydryl and hydroxy, respectively (Figure 1B). Note that there are many serine $(S)$ inside the aerolysin lumen, especially in its sensing regions. To decrease the effects of other amino acids nearby, the K238S is not performed. As shown in Figure 2A, two mutant pores exhibit the almost same open pore current response at biased potential ranging from $0 \mathrm{mV}$ to $+160 \mathrm{mV}$. The open pore currents with standard error (S.D.) of $\sim 2 \mathrm{pA}$ indicate their good structure stability under the experimental conditions. Previous studies have shown that the $(\mathrm{dA})_{4}$ generates the longest translocation durations with the narrowest peak width of blockage current histogram in WT aerolysin among $(\mathrm{dA})_{2}$ to $(\mathrm{dA})_{10}$ (Cao et al., 2016a), which suggests the most stable and uniform behavior during every individual $(\mathrm{dA})_{4}$ translocating through aerolysin. This phenomenon might be related to the optimal spatial filling of $(\mathrm{dA})_{4}$ within the sensing region of aerolysin (Li et al., 2018), leading to the strong interaction between $(\mathrm{dA})_{4}$, and aerolysin lumen. Therefore, we chose $(\mathrm{dA})_{4}$ as the model analyte to study the interaction between nanopore, and oligonucleotide.

Firstly, we statistically analyzed the current signals produced by $(\mathrm{dA})_{4}$ traversing through mutant aerolysin at $+120 \mathrm{mV}$. Our previous study has demonstrated that there are two kinds of events resulting from two dynamic motions of oligonucleotides at the nanopore sensing interface, which are transient bumping events at the pore entrance, and threading events (Cao et al., 2016a). The bumping event originates from the oligonucleotides entering the aerolysin nanopore then eventually returning to the cis solution due to the energy barrier at the entrance, which generates the constant current amplitude with the increasing voltage (Figures S1, S2). We excluded these bumping events in our further statistical analysis since this study focuses on the interaction of an oligonucleotide traversing through the aerolysin. As shown in Figure $2 \mathbf{B}$, the $(\mathrm{dA})_{4}$ experiences the duration time of $3.05 \pm 0.17 \mathrm{~ms}$ inside $\mathrm{K} 238 \mathrm{Y}$, while it sojourns a longer duration time of $29.69 \pm 2.19 \mathrm{~ms}$ at $+120 \mathrm{mV}$ in $\mathrm{K} 238 \mathrm{C}$. The duration time of oligonucleotide is about six times longer than that of WT. Moreover, the oligonucleotides exhibit substantial translocation events inside K238C with Gaussian 


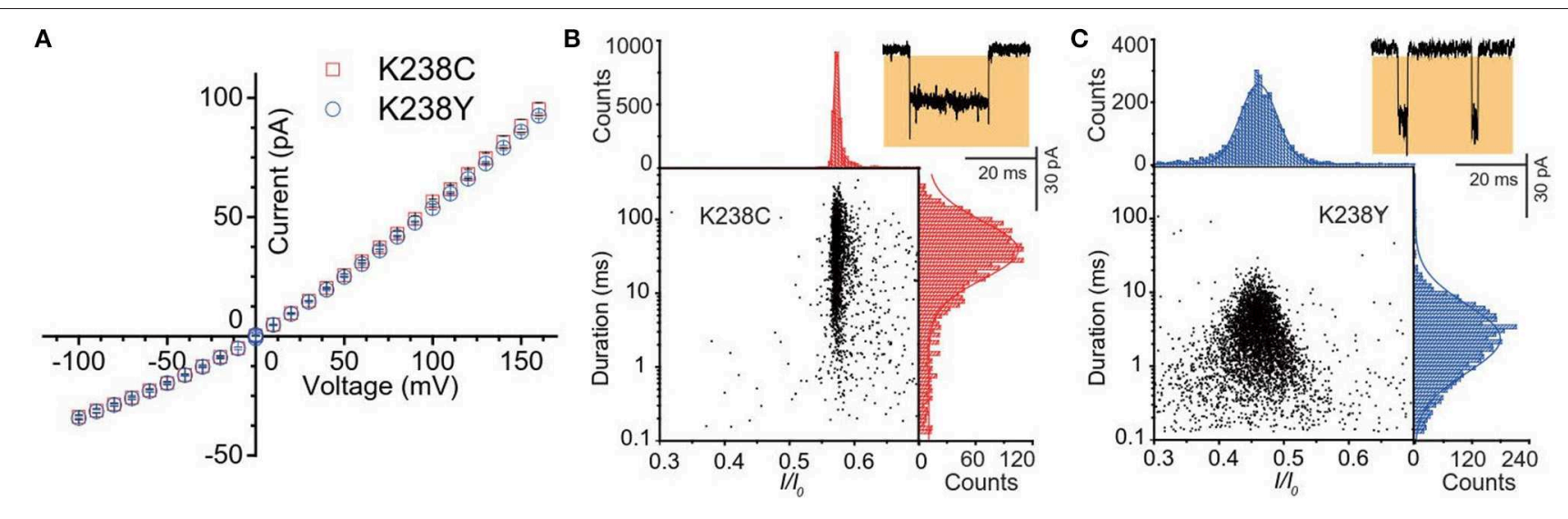

FIGURE 2 | (A) Current-voltage curves of K238C (red) and K238Y (blue). The error-bars are from three independent mutant aerolysin nanopore experiments. Scatter plots of $(\mathrm{dA})_{4}$ along with related $/ / I_{0}$ and duration histograms by $\mathrm{K} 238 \mathrm{C} \mathbf{( B )}$, and $\mathrm{K} 238 \mathrm{Y}(\mathbf{C})$ respectively at $+120 \mathrm{mV}$. $I / I_{0}$ histograms were fitted to single Gaussian function. The log value of duration time histograms were fitted to single Gaussian function.
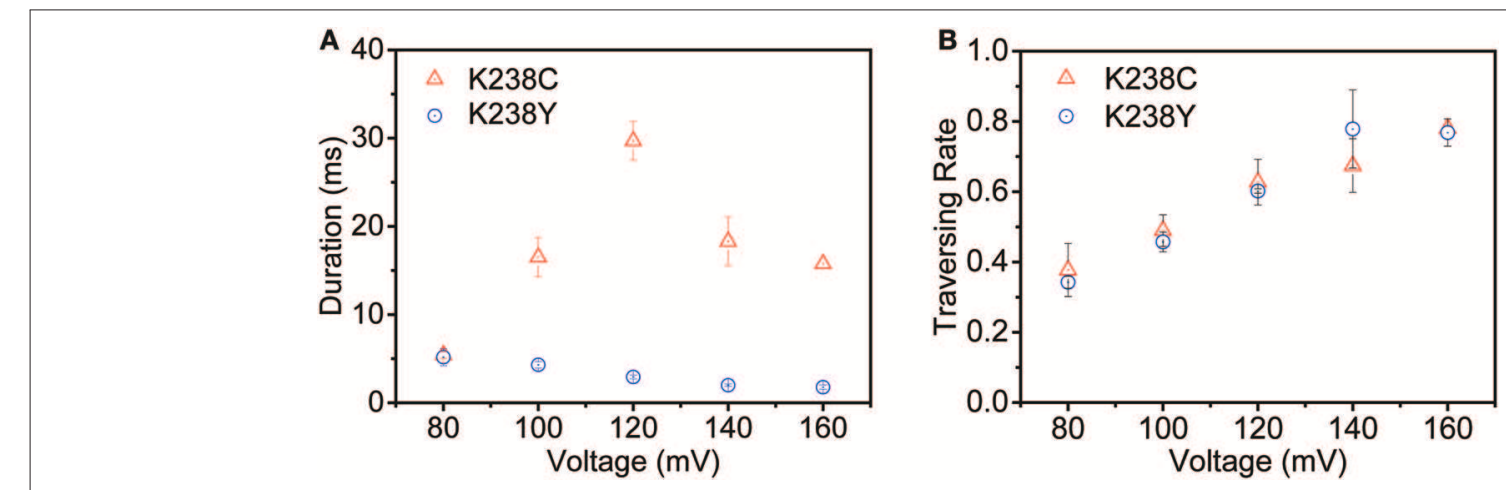

FIGURE 3 | The voltage-dependent of (A) duration time and (B) threading rate plotted against the voltage for (dA) 4 by K238C (red), and K238Y (blue) aerolysin, respectively. The applied voltage ranging from $+80 \mathrm{mV}$ to $+160 \mathrm{mV}$ at $20 \mathrm{mV}$ increments. The error-bars indicated standard deviation from data derived from three independent experiments.

fitting of $I / I_{0}=0.58 \pm 0.01\left(I / I_{0}, I\right.$ represents the residual current, while $I_{0}$ is the open pore ionic current). The full width at half maximum (FWHM) of Gaussian distribution is 0.015 . In contrast, $\mathrm{K} 238 \mathrm{Y}$ produces smaller $I / I_{0}$ value which is concentrated at $0.50 \pm 0.03$ with the FWHM of 0.09 (Figure 2C). The smaller FWHM in K238C suggests a more uniform behavior of $(\mathrm{dA})_{4}$ inside $\mathrm{K} 238 \mathrm{C}$ aerolysin and a stronger interaction between them. Conferring to the previous studies of mutant aerolysin along with WT (Wang et al., 2018a), we have the order of $I / I_{0}$ value as $\mathrm{K} 238 \mathrm{C}>\mathrm{K} 238 \mathrm{G}>\mathrm{WT}>\mathrm{K} 238 \mathrm{Y} \sim \mathrm{K} 238 \mathrm{~F}$. The $I / I_{0}$ value shows an order of side chain volume, which verifies the existence of volume effect. Because the amino acid Y merely have a hydroxyl group difference compared with $\mathrm{F}$, so the $I / I_{0}$ value of $\mathrm{K} 238 \mathrm{Y}$ and $\mathrm{K} 238 \mathrm{~F}$ is almost identical.

The voltage-dependent experiments were then conducted to further understand the non-covalent interaction between pore and analyte. The exponentially decreased duration time with the increasing voltages suggests that $(\mathrm{dA})_{4}$ translocate through the K238Y mutant pore (Figure S3). Moreover, the duration time of $\mathrm{K} 238 \mathrm{Y}$ is lower than that of WT aerolysin at applied potential from $+80 \mathrm{mV}$ to $+160 \mathrm{mV}$, indicating a weaker interaction of $(\mathrm{dA})_{4}$ with tyrosine compared with lysine. However, the K238C exhibits exactly different experimental phenomenon (Figure 3A and Figure S4). When the applied voltage lower than $+120 \mathrm{mV}$, the duration time increases with the increasing voltage. However, when the voltage is higher than $+120 \mathrm{mV}$, the duration time decreases. On the basis of the previous results (Breton et al., 2013), the $+120 \mathrm{mV}$ can be regarded as the threshold voltage $\left(\mathrm{V}_{\mathrm{T}}\right)$ for the translocation of oligonucleotide. If the applied voltage is higher than $\mathrm{V}_{\mathrm{T}}$, the oligonucleotide could traverse through the $\mathrm{K} 238 \mathrm{C}$ mutant pore. More importantly, K238C and K238Y behave similar threading rate which suggest the barrier for the entrance of oligonucleotide into the aerolysin is similar as substituting the amino acid of C and Y at K238 site (Figure 3B). Here, the threading rate represents the ratio of threading events to all the events. Therefore, the evidently different duration time of $(\mathrm{dA})_{4}$ in the $\mathrm{K} 238 \mathrm{C}$, and $\mathrm{K} 238 \mathrm{Y}$ mutant aerolysin indeed depends on the interactions at 238 of aerolysin.

Since K238C and K238Y possess sulfhydryl and hydroxyl, respectively, they are designed to increase the possibility for 
forming hydrogen bonds with the oligonucleotide. As to WT aerolysin, the positively charged lysine is protonated in $\mathrm{pH} 8.0$ solution, which undergoes strong electrostatic interactions with phosphorylate group of oligonucleotides. However, regarding to $\mathrm{K} 238 \mathrm{C}$, the cysteine is a strong hydrogen acceptor, which increases the strength of hydrogen bonds between the pore, and oligonucleotides but weaken the electrostatic interaction in 238 site. Note that the pKa for the sulfydryl of cysteine is about 8.0, suggesting that about half of cysteine should be negatively charged inside the pore at the experimental condition $(\mathrm{pH} 8.0)$. However, the control experiment at $\mathrm{pH} 7.0$ for $(\mathrm{dA})_{4}$ in $\mathrm{K} 238 \mathrm{C}$ aerolysin shows almost same translocation during at each applied voltage (Figure S5), suggesting the same state for sulfydryl

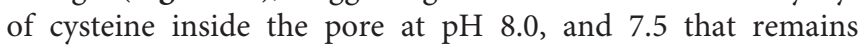
neutral. This result indicates that the $\mathrm{pKa}$ for cysteine inside a confined environment is different from that in bulk conditions. Moreover, our previous study shows that oligonucleotide owns a long duration of $\sim 28.4 \mathrm{~ms}$ in $\mathrm{K} 238 \mathrm{G}$ at $+120 \mathrm{mV}$, which is slightly shorter than that of $\mathrm{K} 238 \mathrm{C}(\sim 29.7 \mathrm{~ms})$. Therefore, the hydrogen bond interaction could enhance the total noncovalent interaction between pore and oligonucleotides, but not the determined effect for the whole translocation process in K238C mutant aerolysin nanopore.

Regarding to $\mathrm{K} 238 \mathrm{Y}$, its side chain contains benzene ring with a hydroxyl. Our previous work has demonstrated that the $\mathrm{K} 238 \mathrm{~F}$ where phenylalanine $(\mathrm{F})$ only contains benzene ring produced a much shorter duration for $(\mathrm{dA})_{4}$. The hydrophobic benzene ring of $\mathrm{K} 238 \mathrm{Y}$ and $\mathrm{K} 238 \mathrm{~F}$ probably repulse the water inside the lumen. Therefore, the amino acid at 238 site for $\mathrm{K} 238 \mathrm{Y}$ and $\mathrm{K} 238 \mathrm{~F}$ is less prone to interact with oligonucleotide. As a result, both $\mathrm{K} 238 \mathrm{Y}$ and $\mathrm{K} 238 \mathrm{~F}$ accelerate the traversing speed of oligonucleotide through aerolysin. Interestingly, the translocation for $\left(\mathrm{dA}_{4}\right)$ in $\mathrm{K} 238 \mathrm{Y}$ is obviously 1.5 times longer than that in K238F (Figure S6), which verify the results that hydrogen bonds is one of the most important factors for the translocation process of oligonucleotides through aerolysin nanopore.

\section{CONCLUSIONS}

In conclusion, our work investigated further into the sensing mechanism for the sensing interface of the aerolysin nanopore. The K238C mutant aerolysin which could form stronger hydrogen bonds with oligonucleotides represented as prolonged

\section{REFERENCES}

Baaken, G., Halimeh, I., Bacri, L., Pelta, J., Oukhaled, A., and Behrends, J. (2015). High-resolution size-discrimination of single nonionic synthetic polymers with a highly charged biological nanopore. Acs Nano 9, 6443-6449. doi: 10.1021/acsnano.5b02096

Balijepalli, A., Ettedgui, J., Cornio, A. T., Robertson, J. W., Cheung, K. P., Kasianowicz, J. J., et al. (2014). Quantifying short -lived events in multistate ionic current measurements. ACS Nano. 8, 1547-1553. doi: 10.1021/nn405761y

Bayley, H., and Cremer, P. S. (2001). Stochastic sensors inspired by biology. Nature 413, 226-230. doi: 10.1038/35093038 duration time compared with $\mathrm{K} 238 \mathrm{G}$ and WT at $+120 \mathrm{mV}$. Similarly, the amino acid tyrosine of K238Y mutation owing to the hydroxyl leads to a longer dwell time than $\mathrm{K} 238 \mathrm{~F}$. These findings determine that the hydrogen bond as one of the important effects attributed to the non-covalent interactions between oligonucleotide and single aerolysin nanopore sensing interface, but not the dominated effect. The complicated analyte-pore interactions including van der Waals, electrostatic interactions, and hydrophobic effect jointly affect translocation behavior. These insights give detailed information for rationally designing the sensing mechanism of the aerolysin nanopore, thereby providing deep understanding for the weak interactions between biomolecules and the confined space for nanopore sensing.

\section{DATA AVAILABILITY}

All datasets generated for this study are included in the manuscript/Supplementary Files.

\section{AUTHOR CONTRIBUTIONS}

M-YL designed and carried out the nanopore experiments, performed image processing, interpreted data, and wrote the manuscript. Y-QW performed proaerolysin production and nanopore experiments, analyzed data, and wrote the manuscript. YL analyzed data, performed image processing, and wrote the manuscript. Y-LY conceived the idea, interpreted data, and wrote manuscript. Y-TL provided substantial contributions to study design, interpreted data, and wrote manuscript.

\section{FUNDING}

This research was supported by the National Natural Science Foundation of China (21834001 and 61871183), Y-LY is sponsored by National Ten Thousand Talent Program for young top-notch.

\section{SUPPLEMENTARY MATERIAL}

The Supplementary Material for this article can be found online at: https://www.frontiersin.org/articles/10.3389/fchem. 2019.00528/full\#supplementary-material

Breton, M. F., Discala, F., Bacri, L., Froster, D., Pelta, J., and Oukhaled, A. (2013) Exploration of neutral versus polyelectrolyte behavior of poly (ethylene glycol) $s$ in alkali ion solutions using single-nanopore recording. J. Phys. Chem. Lett. 4, 2202-2208. doi: 10.1021/jz400938q

Brown, C. G., and Clarke, J. (2016). Nanopore development at Oxford nanopore. Nat. Biotechnol. 34, 810-811. doi: 10.1038/nbt .3622

Butler, T. Z., Pavlenok, M., Derrington, I. M., Niederweis, M., and Gundlach, J. H. (2008). Single-molecule DNA detection with an engineered MspA protein nanopore. Proc. Natl. Acad. Sci. U.S.A. 105, 20647-20652. doi: $10.1073 /$ pnas.0807514106 
Cao, C., Li, M.-Y., Cirauqui, N., Wang, Y.-Q., Dal Peraro, M., Tian, H., et al. (2018). Mapping the sensing spots of aerolysin for single oligonucleotides analysis. Nat. Commun. 9:2823. doi: 10.1038/s41467-018-05 108-5

Cao, C., and Long, Y.-T. (2018). Biological nanopores : confined spaces for electrochemical single-molecule analysis Acc. Chem. Res. 51, 331-341. doi: 10.1021/acs.accounts.7b00143

Cao, C., Ying, Y.-L., Hu, Z.-L., Liao, D.-F., Tian, H., and Long, Y.T. (2016a). Discrimination of oligonucleotides of different lengths with a wild-type aerolysin nanopore. Nat. Nanotechnol. 11, 713-718. doi: 10.1038/nnano.2016.66

Cao, C., Yu, J., Li, M.-Y., Wang, Y. Q., Tian, H., and Long, Y.-T. (2017). Direct readout of single nucleobase variations in an oligonucleotide. Small 13:1702011. doi: 10.1002/smll.201702011

Cao, C., Yu, J., Wang, Y.-Q., Ying, Y.-L., and Long, Y.-T. (2016b). Driven translocation of polynucleotides through an aerolysin nanopore. Anal. Chem. 88, 5046-5049. doi: 10.1021/acs.analchem.6b01514

Deamer, D. W., and Branton, D. (2002). Characterization of nucleic acids by nanopore analysis. Acc. Chem. Res. 35, 817-825. doi: 10.1021/ar000138m

Fahie, M., Chisholm, C., and Chen, M. (2015). Resolved single -molecule detection of individual species within a mixture of anti-biotin antibodies using an engineered monomeric nanopore. ACS Nano. 9, 1089-1098. doi: $10.1021 / \mathrm{nn} 506606 \mathrm{e}$

Forstater, J. H., Briggs, K., Robertson, J. W., Ettedgui, J., Marie-Rose, O., Vaz, C., et al. (2016). MOSAIC: a modular single-molecule analysis interface for decoding multistate nanopore data. Anal. Chem. 88, 11900-11907. doi: 10.1021/acs.analchem.6b03725

Gu, L.-Q., and Shim, J. W. (2010). Single molecule sensing by nanopores and nanopore devices. Analyst. 135, 441-451. doi: 10.1039/B907735A

Iacovache, I., De Carlo, S., Cirauqui, N., Dal Peraro, M., van der Goot, F. G., and Zuber, B. (2016). Cryo-EM structure of aerolysin variants reveals a novel protein fold and the pore-formation process. Nat. Commun. 7:12062. doi: $10.1038 /$ ncomms 12062

Iacovache, I., Degiacomi, M. T., Pernot, L., Ho, S., Schiltz, M., Dal Peraro, M., et al. (2011). Dual chaperone role of the C-terminal propeptide in folding and oligomerization of the pore-forming toxin aerolysin. PLoS Pathog. 7:e1002135. doi: 10.1371/journal.ppat.1002135

Kasianowicz, J. J., Brandin, E., Branton, D., and Deamer, D. W. (1996). Characterization of individual polynucleotide molecules using a membrane channel. Proc. Natl. Acad. Sci. U.S.A. 93, 13770-13773. doi: 10.1073/pnas.93.24.13770

Li, M.-Y., Ying, Y.-L., Fu, X.-X., Yu, J., Liu, S.-C., Wang, Y.-Q., et al. (2018). Resolving the dynamic non-covalent interaction inside membrane protein channel by single-molecule interaction spectrum. ChemRxiv [Preprint]. doi: 10.26434/chemrxiv.7251683.v1

Luscombe, N. M., Laskowski, R. A., and Thornton, J. M. (2001). Amino acid-base interactions: a three-dimensional analysis of protein-DNA interactions at an atomic level. Nucleic Acids Res. 29, 2860-2874. doi: 10.1093/nar/29.13.2860

Mohammad, M. M., Iyer, R., Howard, K. R., McPike, M. P., Borer, P. N., and Movileanu, L. (2012). Engineering a rigid protein tunnel for biomolecular detection. J. Am. Chem. Soc. 134, 9521-9531. doi: 10.1021/ja3043646

Pastoriza-Gallego, M., Rabah, L., Gibrat, G., Thiebot, B., van der Goot, F. G., Auvray, L., et al. (2011). Dynamics of unfolded protein transport through an aerolysin Pore. J. Am. Chem. Soc. 133, 2923-2931. doi: 10.1021/ja10 73245

Pettersen, E. F., Goddard, T. D., Huang, C. C., Couch, G. S., Greenblatt, D. M., Meng, E. C., et al. (2004). UCSF Chimera -a visualization system for exploratory research and analysis. J. Comput. Chem. 25, 1605-1612. doi: $10.1002 /$ jcc. 20084

Piguet, F., Ouldali, H., Pastoriza-Gallego, M., Manivet, P., Pelta, J., and Oukhaled, A. (2018). Identification of single amino acid differences in uniformly charged homopolymeric peptides with aerolysin nanopore. Nat Commun. 9:966 doi: 10.1038/s41467-018-03418-2

Singh, P. R., Bárcena-Uribarri, I., Modi, N., Kleinekathöfer, U., Benz, R., Winterhalter, M., et al. (2012). Pulling peptides across nanochannels: resolving peptide binding and translocation through the hetero-oligomeric channel from nocardia farcinica. ACS Nano. 6, 10699-10707. doi: 10.1021/nn303900y

Soskine, M., Biesemans, A., Moeyaert, B., Cheley, S., Bayley, H., and Maglia, G. (2012). An engineered ClyA nanopore detects folded target proteins by selective external association and pore entry. Nano Lett. 12, 4895-4900. doi: $10.1021 / \mathrm{nl} 3024438$

Stefureac, R., Long, Y.-T., Kraatz, H.-B., Howard, P., and Lee, J. S. (2006). Transport of $\alpha$-helical peptides through $\alpha$-hemolysin and aerolysin pores. Biochemistry. 45, 9172-9179. doi: 10.1021/bi0604835

Wang, G., Wang, L., Han, Y., Zhou, S., and Guan, X. (2013a). Nanopore stochastic detection: diversity, sensitivity, and beyond. Acc. Chem. Res. 46, 2867-2877. doi: 10.1021/ar400031x

Wang, H.-Y., Li, Y., Qin, L.-X., Heyman, A., Shoseyov, O., Willner, I., et al. (2013b). Single-molecule DNA detection using a novel SP1 protein nanopore. Chem. Commun. 49, 1741-1743. doi: 10.1039/c3cc38939a

Wang, Y., Tian, K., Du, X., Shi, R., and Gu, L.-Q. (2017). Remote activation of a nanopore for high performance genetic detection using a ph taxis-mimicking mechanism. Anal. Chem. 89, 13039-13043 doi: 10.1021/acs.analchem.7b03979

Wang, Y., Yao, F. J., and Kang, X.-F. (2015). Tetramethylammonium-filled protein nanopore for single molecule analysis. Anal. Chem. 87, 9991-9997. doi: 10.1021/acs.analchem.5b02611

Wang, Y.-Q., Cao, C., Ying, Y.-L., Li, S., Wang, M.-B., and Long, Y.T. (2018a). Rationally designed sensing selectivity and sensitivity of an aerolysin nanopore via site-directed mutagenesis. ACS Sens. 3, 779-783. doi: 10.1021/acssensors.8b00021

Wang, Y.-Q., Li, M.-Y., Qiu, H., Cao, C., Wang, M.-B., Wu, X.-Y., et al. (2018b). Identification of essential sensitive regions of the aerolysin nanopore for single oligonucleotide analysis. Anal. Chem. 90, 7790-7794. doi: $10.1021 /$ acs.analchem. 8 b01473

Wen, S., Zeng, T., Liu, L., Zhao, K., Zhao, Y., Liu, X., et al. (2011). Highly sensitive and selective DNA-based detection of mercury (II) with $\alpha$-hemolysin nanopore. J. Am. Chem. Soc. 133, 18312-18317. doi: 10.1021/ja206983z

Wendell, D., Jing, P., Geng, J., Subramaniam, V., Lee, T. J., Montemagno, C., et al. (2009). Translocation of double-stranded DNA through membraneadapted phi29 motor protein nanopores. Nat. Nanotech. 4, 765-772. doi: $10.1038 /$ nnano.2009.259

Ying, Y.-L., Cao, C., Hu, Y. X., and Long, Y.-T. (2018). A single biomolecule interface for advancing the sensitivity, selectivity, and accuracy of sensors. Natl. Sci. Rev. 5, 450-452. doi: 10.1093/nsr/nwy029

Ying, Y.-L., Zhang, J., Gao, R., and Long, Y.-T. (2013). Nanopore-based sequencing and detection of nucleic acids. Angew. Chem. Int. Ed. 52, 13154-13161. doi: 10.1002/anie.201303529

Yu, J., Cao, C., and Long, Y.-T. (2017). Selective and sensitive detection of methylcytosine by aerolysin nanopore under serum condition. Anal. Chem. 89, 11685-11689. doi: 10.1021/acs.analchem.7b03133

Conflict of Interest Statement: The authors declare that the research was conducted in the absence of any commercial or financial relationships that could be construed as a potential conflict of interest.

Copyright (c) 2019 Li, Wang, Lu, Ying and Long. This is an open-access article distributed under the terms of the Creative Commons Attribution License (CC BY). The use, distribution or reproduction in other forums is permitted, provided the original author(s) and the copyright owner(s) are credited and that the original publication in this journal is cited, in accordance with accepted academic practice. No use, distribution or reproduction is permitted which does not comply with these terms. 